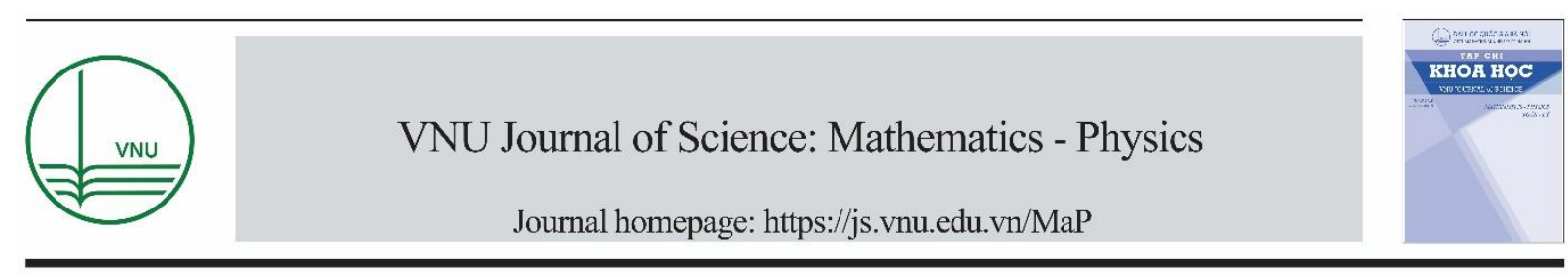

Original Article

\title{
Crystallization Pathway for FeB Nanoparticles
}

\author{
Pham Huu Kien*, Pham Mai An, \\ Nguyen Hong Linh, Giap Thuy Trang
}

Thai Nguyen University of Education, 28 Luong Ngoc Quyen, Thai Nguyen, Vietnam

Received 08 April 2019

Revised 04 June 2019; Accepted 18 July 2019

\begin{abstract}
In this study, the FeB nanoparticle (NP) consisting of 5,000 particles (4,500 $\mathrm{Fe}$ atoms and $500 \mathrm{~B}$ atoms) was investigated by means of molecular dynamics (MD) simulation. When the amorphous $\mathrm{FeB}$ nanoparticle is annealed at the temperature of $900 \mathrm{~K}$ for a long time, it is crystallized into a bcc crystalline structure. The simulation shows that the sample undergoes crystallization via the nucleation mechanism. During the crystallization, B atoms diffuse to the boundary region of $\mathrm{Fe}$ crystal. The crystal growth proceeds when this boundary region attains specific properties which are defined by the fraction of $\mathrm{B}$ atoms and the energies of $\mathrm{AB}$-atoms and $\mathrm{CB}$-atoms. The study further indicates that the crystalline and mixed FeB nanoparticle consists of three distinct parts including $\mathrm{Fe}$ crystalline and two FeB amorphous parts (B-poor and B-rich amorphous parts). The different polymorphs of FeB nanoparticle differ in the local structure, size of Fe crystal and energies of different type atoms.
\end{abstract}

Keywords: Annealing, B-poor, B-rich, crystal, amorphous, polymorphs.

\section{Introduction}

The understanding of physical properties of nanomaterials of amorphous and crystalline nature is the goal of huge research activity during last decades [1-5]. The nanomaterials can be obtained in different shapes and polymorphic structures depending on production methods [3, 6-9]. Since in 1911, Fe precipitates were already obtained by the chemical procedures [7]. More recently, the NP has been synthesized by chemical reduction synthesis [8] which enables to produce not only particles with a simple set-up, but also to obtain metallic glasses. Regarding magnetic materials, it is easy to see the interest provoked by so-called "nanocomposite" systems [2] which consist of two or more different

\footnotetext{
${ }^{*}$ Corresponding author.

Email address: phkien80@ gmail.com

https//doi.org/ 10.25073/2588-1124/vnumap.4346
} 
phases. These systems at the nanometer scale give rise to surprising effects. For examples, spherical Co NP with shell/core structure allows beating the super-paramagnetic limit. The shell/core M-B NP $(\mathrm{M}=\mathrm{Fe}, \mathrm{Co})$ with amorphous, mixed amorphous and bcc Fe were obtained using the chemical reduction of metallic salts by sodium borohydride [10,11]. The structural arrangement of atoms is analyzed through EXANES and EXAFS. It was shown that the presence of a number of bcc crystals increases the particle coercively. More detail about the structure and physical properties of the materials can be obtained from simulations [12-19]. Molecular dynamics simulation is a powerful tool to explore the local structure and the atomistic behavior of interfaces between different phases coexisted in the system. Hence, the present work is devoted to investigate the local structure of $\mathrm{FeB}$ NP polymorphs included the amorphous, crystalline and mixed samples.

Amorphous state of NP generally is unstable, and amorphous NP will be crystallized upon appropriate annealing. The stability of amorphous NPs against crystallization plays an important role because of this related to their working ability in practice. The crystallization of amorphous NPs is studied intensively by experiments [20-26]. It was shown that the crystallization in NP proceeds via the nucleation, but exhibits certain specific features comparing to the bulk counterpart. However, the crystallization mechanism at the atomic level in this material has remained unclear yet. So the interest of present work is twofold: to clarify the local structure of NP polymorphs, and secondary, to observe how the crystallization happens in FeB NPs. Especially, the role of B atoms that prevent the formation and growth of crystal, is also the goal of present work. For this, we have prepared amorphous FeB sample at $300 \mathrm{~K}$ and $900 \mathrm{~K}$. The specific annealing procedures have done to obtain the crystalline and mixed samples.

\section{Calculation procedure}

To obtain proper result from MD simulation, the choice of inter-atom potentials is most important. It is interesting that the simple potential proposed by Pak and Doyama long time ago, well describes thermodynamics and structure properties of $\mathrm{Fe}$ and Fe-alloys materials. MD simulations carried out by various researchers and using Pak-Doyama potential confirmed these points [27-34]. Therefore, in the present work, we conduct the MD simulation using Pak-Doyama type potentials to describe the interaction between atoms in NP samples. The form of this potential is given follow [31-34]:

$$
U(r)=\left\{\begin{array}{lr}
a(r+b)^{4}+c(r+d)^{2}+e, & 0 \leq r \leq r_{\text {cutoff }} \\
0 & r_{\text {cutoff }}<r
\end{array}\right.
$$

where $r$ is the inter-atomic distance and in $\AA, U(r)$ is in $\mathrm{eV}$. The parameters of potentials (1) are given in Table 1.

Table 1. The parameters of potential

\begin{tabular}{lllllll}
\hline Pairs & $a\left(\mathrm{eV} / \AA^{4}\right)$ & $b(\AA)$ & $c\left(\mathrm{eV} / \AA^{2}\right)$ & $d(\AA)$ & $c(\mathrm{eV})$ & $r_{\text {cutoff }}(\AA)$ \\
\hline Fe-Fe & -0.18892 & -1.82709 & 1.70192 & -2.50849 & -0.19829 & 3.44 \\
Fe-B & -0.22407 & -1.47709 & 2.01855 & -2.15849 & -0.23519 & 3.09 \\
B-B & -0.08772 & -2.17709 & 0.79028 & -2.85849 & -0.09208 & 3.79 \\
\hline
\end{tabular}

The MD simulation is performed for a system containing 5000 atoms with free boundary conditions. The equations of motion were solved numerically using the Verlet algorithm. The MD step 
is equal to $0.46 \mathrm{fs}$. Initially, all atoms including $4500 \mathrm{Fe}$ atoms and $500 \mathrm{~B}$ atoms, are randomly placed in a sphere with radii of $28.5 \AA$. Then the statistical relaxation is carried out until the system reached the equilibrium. This sample was heated to $300 \mathrm{~K}$. The obtained sample then has relaxed isothermally (annealing) by $8 \times 10^{7}$ steps to prepare the amorphous NP sample. By this way, FeB NP sample which contains $10 \% \mathrm{~B}$ atoms has been constructed. This well-equilibrated sample is called FeB 300 -sample. To study the crystallization we have prepared 900 -sample by heating the 300 -sample to $900 \mathrm{~K}$ and then relaxing isothermally over $10^{7}$ steps. To analyze the atomistic arrangement of Fe atoms in NP we determine the pair radial distribution function (PRDF) for Fe-Fe pair using the procedure reported in the previous work [27,34].

In Fig.1 schematically illustrates a mixed sample of NP. There is a crystal cluster inside the amorphous matrix. For the convenience, the atoms belonging to amorphous and crystalline phases are called Am-atom and Cr-atom, respectively. During the annealing Am-atoms and Cr-atoms may be transited from one to another type. In following we denote $N_{A m}$ and $N_{C r}$ to the number of Am-atoms and $\mathrm{Cr}$-atoms, respectively. There is a boundary region between amorphous and crystalline phases. The $\mathrm{Am}$-atoms, $\mathrm{Cr}$-atoms in this region are called AB-atom, CB-atom, respectively. The Am-atoms and $\mathrm{Cr}$-atoms located outside the boundary region are called AV-atom and $\mathrm{CV}$-atom, respectively. $\mathrm{We}$ denote $N_{A B}, N_{A V}, N_{C B}$ and $N_{C V}$ to the number of AV-atoms, AB-atoms, CB-atoms and CV-atoms, respectively. Obviously $N_{C r}=N_{C B}+N_{C V} ; N_{A m}=N_{A V}+N_{A B}$. To determine a particular atom be Cr-atom or Am-atom, a following criterion was applied. That is, the Fe atom was identified as having the bcc configuration if it satisfies two conditions: (i) having 14 Fe neighbors; (ii) six among these neighbors have 4 neighbors and remaining ones have 6 neighbors in common with the given atom. The cutoff radius to determine the neighboring atom is equal to $3.35 \AA$. Such Fe atom and its 14 neighbors belong to Cr-atoms and form a basic nucleus of bcc crystal. Two basic nuclei are linking if they have at least a common Cr-atom. A crystal cluster consists of basic nuclei that each nucleus links at least to another nucleus of the cluster.

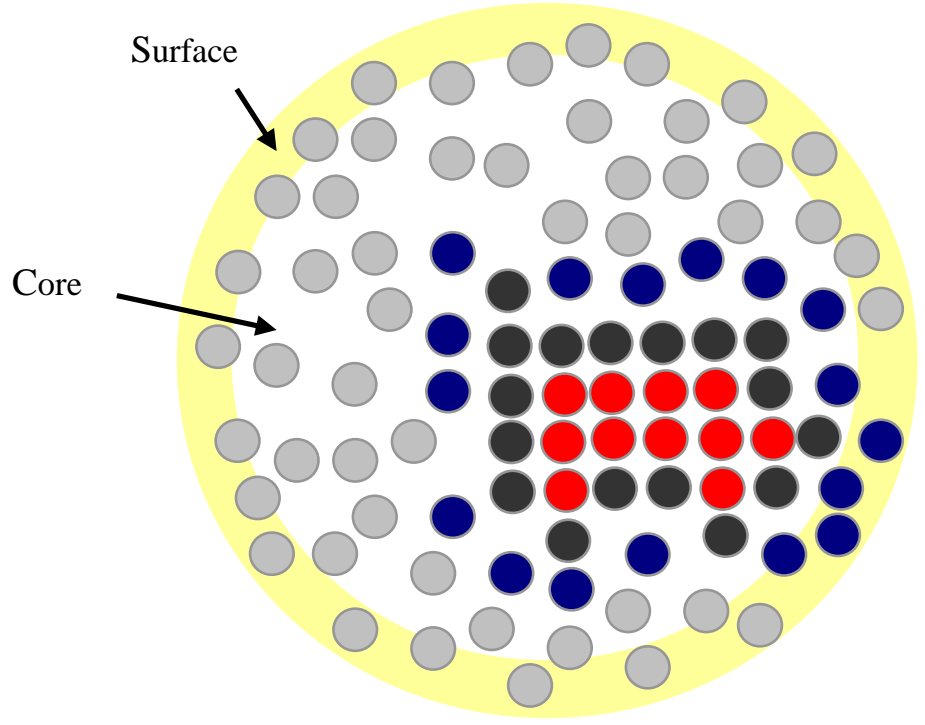

Fig. 1. Schematic illustration of a mixed sample of NP: blue, black, grey and red circles represent AB-atom, $\mathrm{CB}$-atom, $\mathrm{AV}$-atom and $\mathrm{CV}$-atoms, respectively. 


\section{Results and discussion}

It is still interesting that although the crystallization of $\mathrm{Fe}_{100-\mathrm{x}} \mathrm{B}_{\mathrm{x}} \mathrm{NPs}$, where $x=0,2,4,5$ and 10 have been studies in the previous works [32-34], we still have not been considered the role of the B atoms preventing the formation and growth of crystal in $\mathrm{Fe}_{90} \mathrm{~B}_{10} \mathrm{NP}$, as well as how are the amorphous, crystalline and mixed phases formed?. This is also main purpose of the present work. In this paper, the nucleation and crystal growth have been identified through the time evolution of crystal cluster forming in NP and mean potential energy per atom. In Fig.2 we show the number of Cr-atoms $N_{C r}$ for 900 -samples as a function of times. The process can be divided into three periods. In the first period there are only fewer nuclei which form and dissolve quickly. $N_{C r}$ detected within this period is close to zero. Meanwhile, in the second period $N_{C r}$ significantly increases indicating that a crystal cluster forms and it substantially grows with times. In the third period, $N_{C r}$ fluctuates around a saturation value and the crystal growth completes.

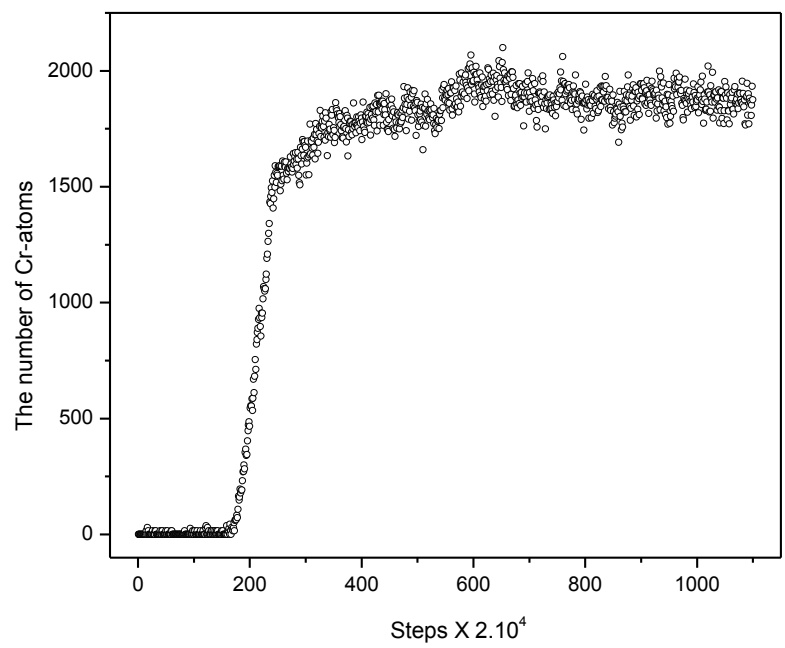

Fig. 2. The time dependence of the number of $\mathrm{Cr}$-atoms.

The crystallization process can be viewed directly through the 3D-image of atomic arrangement. Here we extract some representative snapshots of NP detected at different moments during the process. As shown in Fig.3 shows the spatial distribution of CV-atoms recorded for different time intervals during annealing process. Note that at present moment only small amount of $\mathrm{CV}$-atoms presented in Fig.3, is in the "state of Cr-atom", remaining ones in this state at early moment. For initial stage of crystallization CV-atoms distribute uniformly in the NP. In the next time interval there is a number of $\mathrm{CV}$-atoms which located nearby. These atoms have life time much longer than other $\mathrm{CV}$-atoms. For the time interval shown in Fig.3E, most CV-atoms appear inside a small volume of NP. It means that as a cluster with critical size appears, new $\mathrm{CV}$-atoms appear most frequently in the boundary region of crystal (BRC). In summary, the simulation result shows that small nuclei frequently form and disappear at the initial stage of crystallization. After long annealing times the structure of NP changes so that some $\mathrm{CV}$-atoms have the life time much longer than other atoms. This supports to forming a cluster with specific boundary. As shown in Fig.4, a crystal cluster containing 178 atoms is found at the step $n_{l}$. After annealing by $5 \times 10^{5}$ steps this cluster grows to reach the size $N_{C r}=278$ atoms. Next $5 \times 10^{5}$ steps this cluster grows to reach the size $N_{C r}=424$ atoms. Further annealing increases $N_{C r}$ to 628 atoms. 
A)

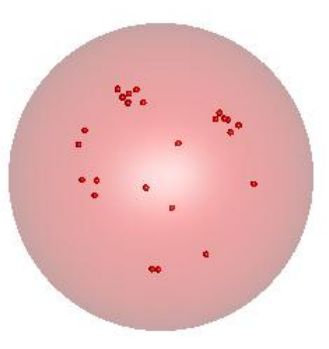

B)

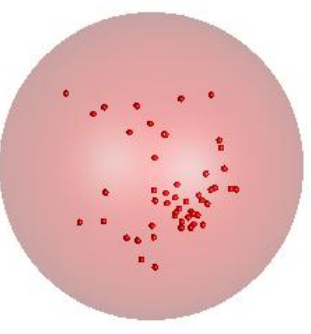

C)

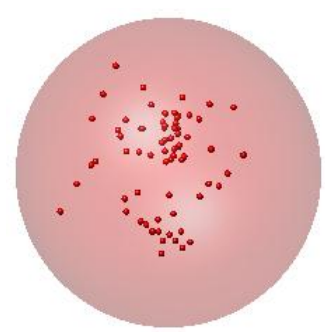

D)

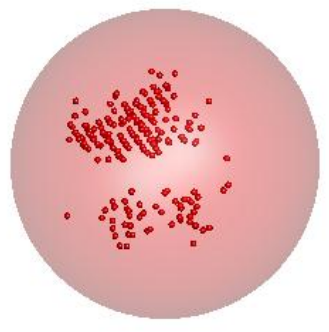

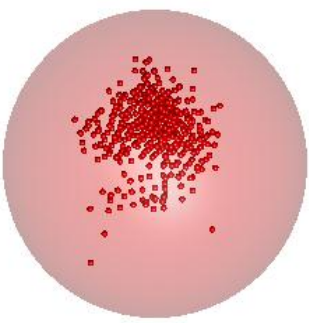

E)

Fig. 3. The spatial distribution of $\mathrm{Cr}$-atoms in the FeB sample which are recorded for different time interval under annealing. A, B, C) initial stage of formation of nuclei which are disappeared quickly; D) the nuclei form nearby and a small cluster appears; E) a new crystal cluster is created and grows.

(A

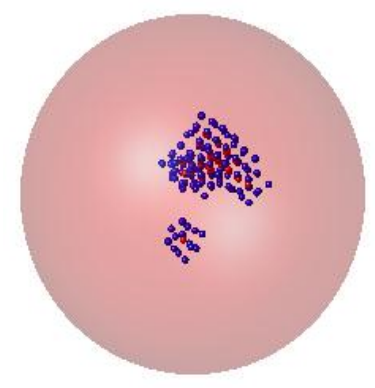

$(\mathrm{C}$

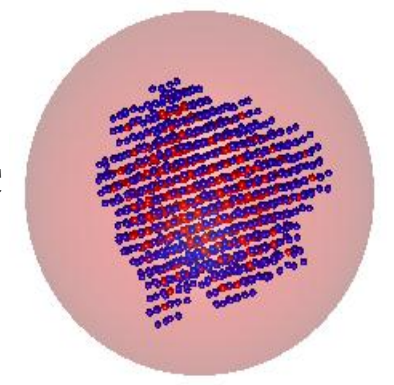

(B)

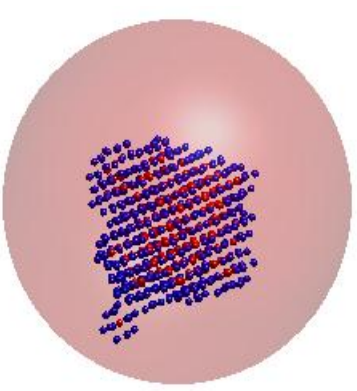

$(\mathrm{D}$

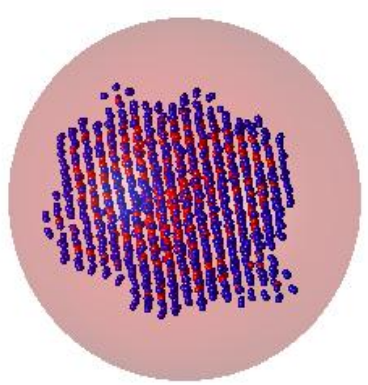

Fig.4. Snapshot of Cr-atom arrangement in the FeB sample at: (A) steps $n_{1}, N_{C r}=178$; (B) steps $n_{1}+5 \times 10^{5}$, $N_{C_{r}}=278$; (C) steps $n_{l}+1 \times 10^{6}, N_{C_{r}}=424$; (D) steps $n_{l}+1.5 \times 10^{6}, N_{C_{r}}=627$. 
Fig. 5 shows the time dependence of number of atoms and the fraction of B atoms during $n_{1}=5 \times 10^{6}$ steps. The number of bcc Fe crystal increases to 2300 . The number of atoms in I- amorphous $\mathrm{FeB}$ phase is larger than that in II-amorphous FeB phase. This result is due to the different B atom fraction. It means that B atom fraction significantly effects to crystallization process. Fig. 6 shows the time dependence of mean energy per atom for different type atoms of FeB sample. In the case of the large cluster the mean potential energy per atom of $\mathrm{CV}$-atoms and $\mathrm{CB}$ - atoms is much less than that of $A V$ atoms. Hence, the transition from amorphous-atom to crystal-atom proceeds more frequently than the transition from crystal-atom to amorphous-atom. As a result, the forming crystal cluster is stable and tends to grow with time. It can be seen that the crystalline process is very close to classical crystallization theory and Ostwald's step rule.

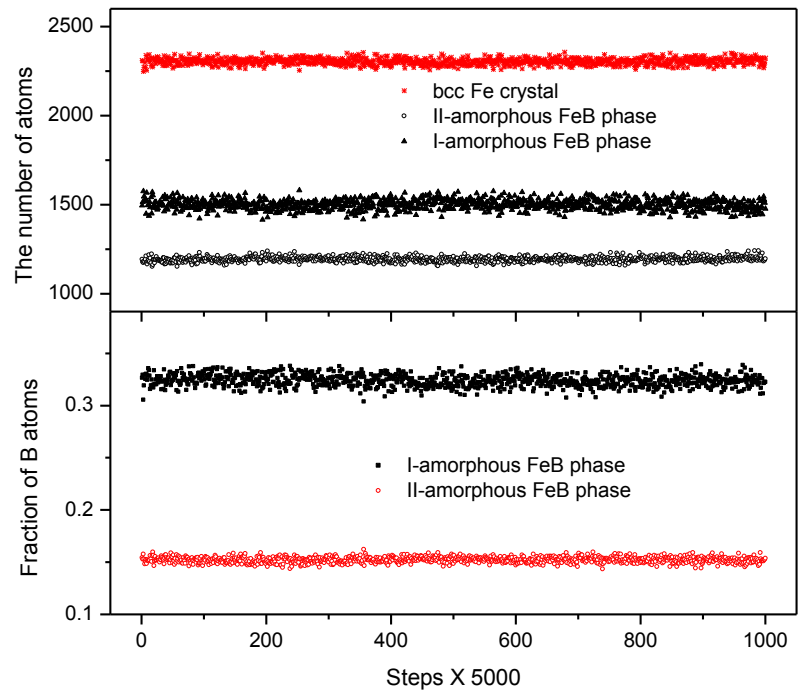

Fig.5. The time dependence of number of atoms and fraction of B atoms for FeBsample.

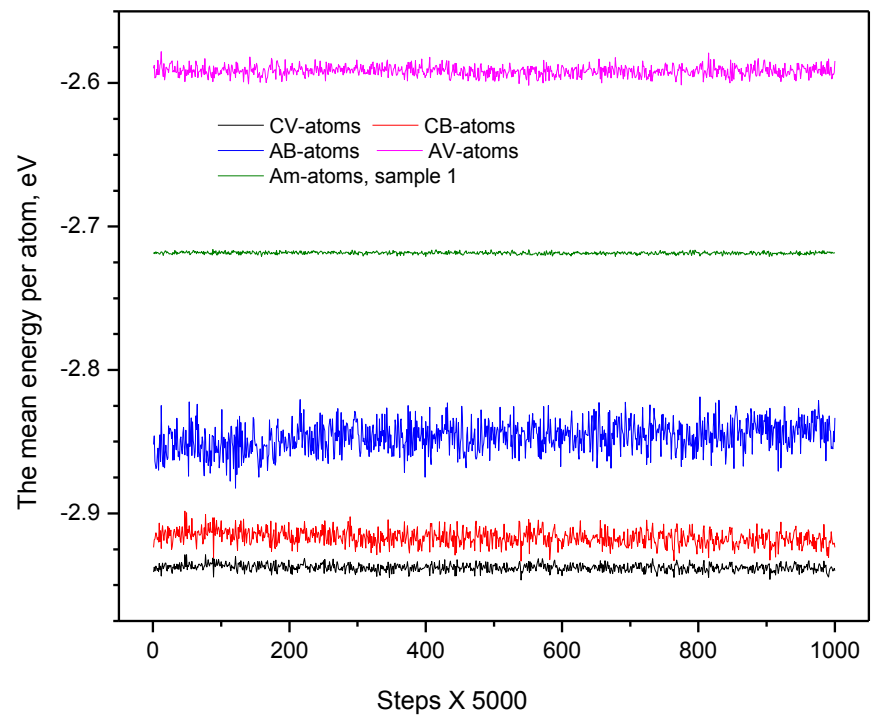

Fig.6. The time dependence of mean energy per atom for different type atoms of FeB sample. 


\section{Conclusion}

In this paper, the annealing of $\mathrm{Fe}_{90} \mathrm{~B}_{10} \mathrm{NP}$ at temperature of $900 \mathrm{~K}$ has been simulated. Several results are demonstrated as follows

i) The crystallization happens when the $\mathrm{Fe}_{90} \mathrm{~B}_{10} \mathrm{NP}$ is heated to $900 \mathrm{~K}$ and relaxed for long times. During the crystallization, B atoms move out places where the crystal locates, and diffuse to the boundary region. The crystalline process is very close to classical crystallization theory and Ostwald's step rule.

ii) Regarding the polymorphs of FeB NP, we have considered the amorphous, crystalline and mixed NP samples. The result shows that the mixed and crystalline sample consists of three distinct parts including a Fe bec crystalline and two FeB amorphous parts (B-poor and B-rich amorphous parts). We found that these parts for different polymorphs differ strongly in the local structure, the concentration of B and energies of different type atoms. In there, the B atoms prevent the formation and growth of crystal.

\section{Acknowledgement}

This research is funded by Vietnam National Foundation for Science and Technology Development (NAFOSTED) under grant number 103.02-2018.312.

\section{References}

[1] Y. Shibuta, Y. Watanabe, T. Suzuki, Growth and melting of nanoparticles in liquid iron: A molecular dynamics study, Chemical Physics Letters 475 (2009) 264-268.

[2] E.V. Levchenko, A.V. Evteev, D.P. Riley, I.V. Belova, G.E. Murch, Molecular dynamics simulation of the alloying reaction in Al-coated Ni nanoparticle, Computational Materials Science 47 (2010) 712-720.

[3] V.V. Hoang, D. Ganguli, Amorphous nanoparticles-Experiments and computer simulations, Physics Reports 518 (2012) 81-140.

[4] K.J. Carroll, J.A. Pitts, Kai Zhang, A. K. Pradhan, E. E. Carpenter, Nonclassical crystallization of amorphous iron nanoparticles by radio frequency methods, Journal of Applied Physics 107 (2010) 09A302.

[5] S. Qin, W. Lei, D. Liu, P. Lamb, Y. Chen, Synthesis of single-crystal nanoparticles of indium oxide by "urea glass" method and their electrochemical properties, Materials letters 91 (2013) 5-8.

[6] R.C. O’Handley, Modern Magnetic Materials: Principles and Applications, Wiley, New York, 2000.

[7] A.L. Oppegard, F.J. Darnell, H.C. Miller, Magnetic properties of single-domain iron and iron-cobalt particles prepared by borohydride reduction, Journal of applied physics 32 (1961) S184-S185.

[8] J. van Wonterghem, S. Morup, C.J.W. Koch, S.W. Charles, S. Wells, Formation of ultra-fine amorphous alloy particles by reduction in aqueous solution, Nature 322 (1986) 622.

[9] A. Yedra, et al., Survey of conditions to produce metal-boron amorphous and nanocrystalline alloys by chemical reduction, Journal of non-crystalline solids 287 (2001) 20-25.

[10] V. Skumryev, S. Stoyanov, Y. Zhang, G. Hadjipanayis, D. Givord, J. Nogues, Beating the superparamagnetic limit with exchange bias, Nature 423 (2003) 850-861.

[11] Q.A. Pankhurst, et al., Interfacial exchange pinning in amorphous iron-boron nanoparticles, Physical Review B 69 (2004) 212401.

[12] Z.Y. Hou, L.X. Liu, R.S. Liu, Simulation study on the evolution of thermodynamic, structural and dynamic properties during the crystallization process of liquid Na, Modelling and Simulation in Materials Science and Engineering 17 (2009) 035001. 
[13] J.H. Shim, S.C. Lee, B.J. Lee, J.Y. Suh, Y.W. Cho, Molecular dynamics simulation of the crystallization of a liquid gold nanoparticle, Journal of Crystal Growth 250 (2003) 558-564.

[14] V.V. Hoang, N.H. Cuong, Local icosahedral order and thermodynamics of simulated amorphous Fe, Physica B: Condensed Matter 404 (2009) 340-346.

[15] X. Li, J. Huang, Molecular dynamics studies of the kinetics of phase changes in clusters III: structures, properties, and crystal nucleation of iron nanoparticle Fe331, Journal of Solid State Chemistry 176 (2003) 234-242.

[16] J.J. Chu, C.A. Steeves, Thermal expansion and recrystallization of amorphous Al and Ti: A molecular dynamics study, Journal of Non-Crystalline Solids 357 (2011) 3765-3773.

[17] C.B.B. Costa, R.M. Filho, Nanoparticle processes modelling: The role of key parameters for population balances for on-line crystallization processes applications, Powder Technology 202(2010) 89-94.

[18] M. Karaman, M. Aydın, S.H. Sedani, K. Ertuk, R. Turan, Low temperature crystallization of amorphous silicon by gold nanoparticle, Microelectronic Engineering 108 (2013) 112-115.

[19] S. Jungblut, C. Dellago, Crystallization of a binary Lennard-Jones mixture, The Journal of chemical physics 134 (2011) 104501.

[20] H.V. Hue, Crystallization of Amorphous Iron Nano-particles by Means of Molecular Dynamics Simulation, Int J Nano Stud Technol 4 (2015) 88-92.

[21] M. Alcoutlabi, G.B. McKenna, Effects of confinement on material behaviour at the nanometre size scale, Journal of Physics: Condensed Matter 17 (2005) 461-470.

[22] L. Gao, Q. Zhang, Effects of amorphous contents and particle size on the photocatalytic properties of $\mathrm{TiO}_{2}$ nanoparticles, Scripta materialia 44 (2001) 1195-1198.

[23] H. Zhang, J.F. Banfield, Kinetics of crystallization and crystal growth of nanocrystalline anatase in nanometersized amorphous titania, Chemistry of materials 14 (2002) 4145-4154.

[24] G. Madras, B.J. McCoy, Kinetic model for transformation from nanosized amorphous $\mathrm{TiO}_{2}$ to anatase, Crystal growth \& design 7 (2007) 250-253.

[25] C. Pan, P. Shen, S.Y. Chen, Condensation, crystallization and coalescence of amorphous $\mathrm{Al}_{2} \mathrm{O}_{3}$ nanoparticles, Journal of crystal growth 299 (2007) 393-398.

[26] M. Epifani, E. Pellicer, J. Arbiol, N. Sergent, T. Pargnier, J.R. Morante, Capping ligand effects on the amorphous-to-crystalline transition of CdSe nanoparticles, Langmuir 24 (2008) 11182-11188.

[27] P.H. Kien, M.T. Lan, N.T. Dung, P.K. Hung, Annealing study of amorphous bulk and nanoparticle iron using molecular dynamics simulation, International Journal of Modern Physics B 28 (2014) 1450155.

[28] A.V. Evteev, A.T. Kosilov, E.V. Levchenko, O.B. Logachev, Kinetics of isothermal nucleation in a supercooled iron melt, Physics of the Solid State 48 (2006) 815-820.

[29] M. Hirano, K. Shinjo, Atomistic locking and friction, Physical Review B 41 (1990) 11837.

[30] T.P. Duy, V.V. Hoang, Atomic mechanism of homogeneous melting of bcc $\mathrm{Fe}$ at the limit of superheating, Physica B: Condensed Matter 407 (2012) 978-984.

[31] P.K. Hung, L.T. Vinh, P.H. Kien, About the diffusion mechanism in amorphous alloys, Journal of NonCrystalline Solids 356 (2010) 1213-1216.

[32] P.H. Kien, N.T. Thao, P.K. Hung, The local structure and crystallization of FeB nanoparticle, Modern Physics Letters B 28 (2014) 1450246.

[33] Kien Pham Huu, Trang Giap Thi Thuy, Hung Pham Khac, The study of separation of crystal Fe and morphology for FeB nanoparticle: Molecular dynamics simulation, AIP Advances 7 (2017) 045301.

[34] P.H. Kien, P.K. Hung, N.T. Thao, Molecular dynamic simulation of Fe nanoparticles, International Journal of Modern Physics B 29 (2015) 1550035. 